\title{
La búsqueda de información, la selección y creación de contenidos y la comunicación docente
}

\section{(The search for information, the selection and creation of contents and teacher communication)}

\author{
Juan Luis Cabanillas García \\ Ricardo Luengo González \\ José Luis Torres Carvalho \\ Universidad de Extremadura (España)
}

DOI: $\underline{\text { http://dx.doi.org/10.5944/ried.23.1.24128 }}$

\section{Cómo referenciar este artículo:}

Cabanillas García, J. L., Luengo González, R., y Torres Carvalho, J. L. (2020). La búsqueda de información, la selección y creación de contenidos y la comunicación docente. RIED. Revista Iberoamericana de Educación a Distancia, 23(1), pp. 241-267. doi: http://dx.doi.org/10.5944/ried.23.1.24128

\section{Resumen}

La sociedad de la información y del conocimiento ha crecido a pasos agigantados en la docencia a partir del auge de la educación a distancia y la inclusión de las TIC. Esto implica que los docentes deben ser, y sentirse digitalmente competentes para poder desarrollar su labor de forma eficaz. Hemos desarrollado un estudio para analizar tres de los parámetros que definen la competencia digital, en docentes de la provincia de Badajoz: el tratamiento de la información, la selección y creación de contenidos y la comunicación docente. Junto a estas variables, hemos incluido parámetros sociodemográficos que nos ayuden a definir cuáles son claves dentro de la configuración de la competencia digital docente, como son el género, la edad, la experiencia, la titulación académica máxima, el nivel educativo de docencia y la población del municipio de docencia. Los resultados han indicado que, prioritariamente, el profesorado otorga mayor importancia al tratamiento de la información y que, tanto la edad como la titulación académica del docente, son variables fundamentales, ya que, a medida que aumenta la edad y mejora la formación del docente, mejoran las variables que configuran la competencia digital, capacitando al profesorado para una labor docente correcta en términos digitales y, por ello, más adaptados a las necesidades de la escuela contemporánea.

Palabras clave: alfabetización informática; tecnologías de la información y dela comunicación; información; comunicación; aprendizaje asistido por ordenador. 


\begin{abstract}
The information and knowledge society has grown by leaps and bounds from the impulse of the distance education and the inclusion of the ICT in teaching. This implies that teachers must be and feel digitally competent to develop their work effectively. We have developed a study to analyze three parameters that define the digital competence in teachers of the province of Badajoz: the treatment of information, the selection and creation of content and teacher communication. Together with these variables, we have included sociodemographic parameters that help us to define the keys within the configuration of the digital teaching competence, such as the gender, the age, the experience, the máximun academic degree, the educational level of teaching and the population of the teaching municipality. The results have shown as a priority, teachers give greater importance to the treatment of information and that both the age and the academic qualifications of the teacher are fundamental variables, since as the age increases and the teacher's training improves, the variables that configure the digital competence, training teachers for a correct teaching activity in digital terms, and therefor, more adapted to the needs of the contemporary school.
\end{abstract}

Keywords: computer literacy; information and communication technologies; information; communication; computer-assisted learning.

La competencia digital es una de las competencias que ha adquirido más importancia durante la última década, siendo integrada en la mayor parte de los currículos de todas las etapas. Concretamente, en la Ley Orgánica 2/2006 de 3 de mayo (LOE) modificada por la Ley Orgánica 8/2013 de 9 de diciembre para la Mejora de la Calidad Educativa (LOMCE), establece que es una de las competencias clave que debe adquirir el alumnado, con las siguientes referencias en los currículos de cada una de las etapas:

- Real Decreto 126/2014, de 28 de febrero, por el que se establece el currículo básico de la Educación Primaria, que, en su artículo 2, menciona la competencia digital como una de las competencias claves a adquirir por el alumnado de Educación Primaria.

- Real Decreto 1105/2014, de 26 de diciembre, por el que se establece el currículo básico de la Educación Secundaria Obligatoria y el Bachillerato, que, de igual modo, en su artículo 2, menciona la competencia digital como una de las competencias clave a adquirir por el alumnado de Educación Secundaria Obligatoria y el Bachillerato.

- Real Decreto 1147/2011, de 29 de julio, por el que se establece la ordenación general de la formación profesional del sistema educativo, que muestra en su anexo II y III el tratamiento de la información y competencia digital como una de las competencias mínimas que debe ser alcanzada, tanto por el alumnado de ciclos de grado medio como grado superior. 


\section{CONTEXTUALIZACIÓN DE LA COMPETENCIA DIGITAL DOCENTE}

A nivel Europeo, UNESCO (2008) establece un marco de referencia que reflejamos en la figura 1, que permita a los docentes vincular la formación reglada con el fin de alcanzar los objetivos politicos y económicos.

Figura 1. Estándares UNESCO de competencias TIC para docentes

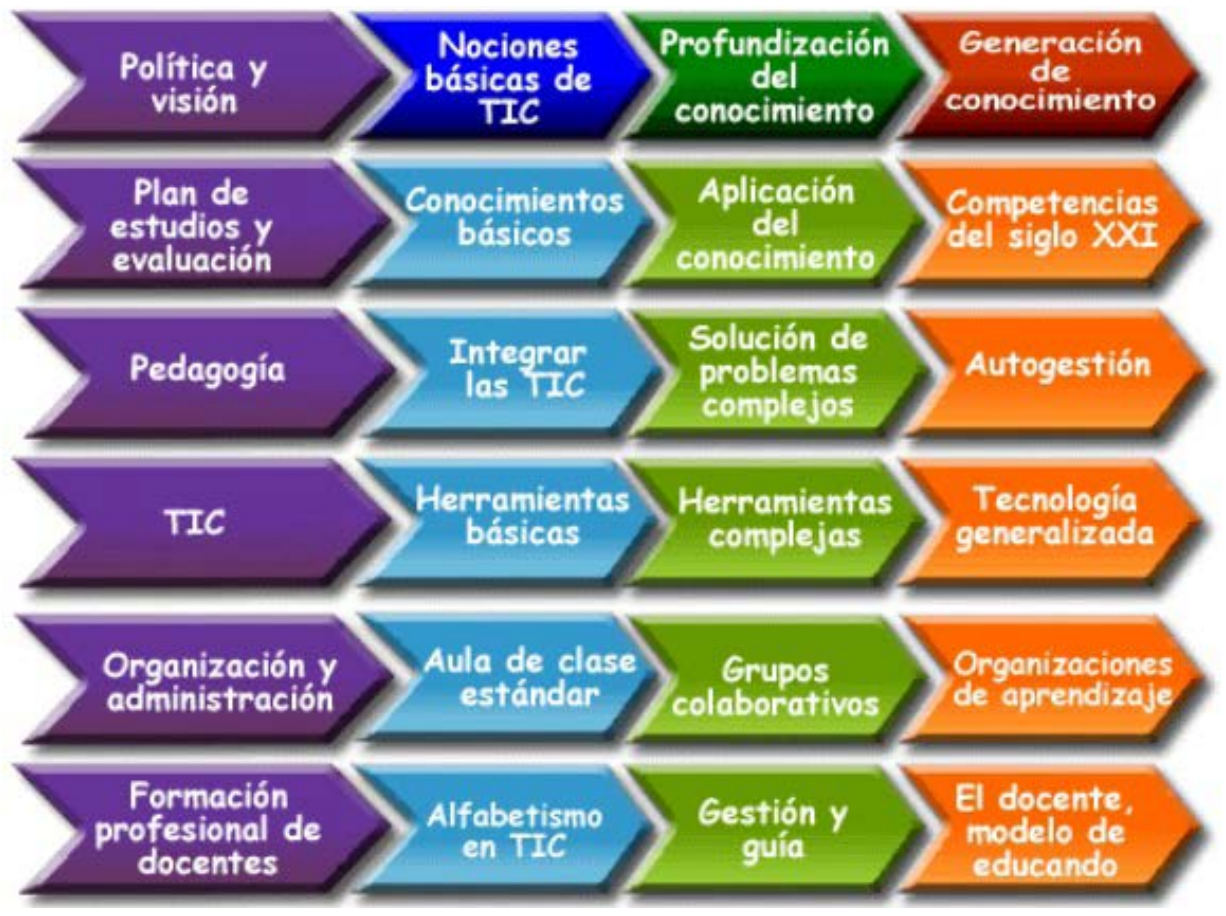

Fuente: UNESCO (2008).

El nivel de nociones básicas en TIC incluye competencias que permiten poder usar metodologías educativas existentes, como juegos, contenidos de Internet, laboratorios de informática o recursos que permitan utilizar las TIC para alcanzar los objetivos curriculares. El nivel de profundización en el conocimiento supone elevar la capacidad del alumnado, agregando valor a la sociedad y a la economía para resolver problemas complejos que suceden en situaciones reales de la vida. En este enfoque, los docentes deben comprender los objetivos en materia de políticas educativas y las prioridades sociales. El último nivel de generación del conocimiento implica incrementar la productividad, desarrollando estudiantes, ciudadanos y trabajadores que puedan generar conocimiento, innovar y aprender a lo largo de su vida. 
En una posterior revision, UNESCO (2011) nos muestra los seis aspectos que definen el trabajo docente para el desarrollo de un adecuado aprendizaje del uso y conocimiento de los recursos TIC para poder desarrollar una óptima competencia digital. (figura 2).

Figura 2. Los 6 aspectos que definen el trabajo docente para la mejora de la competencia digital

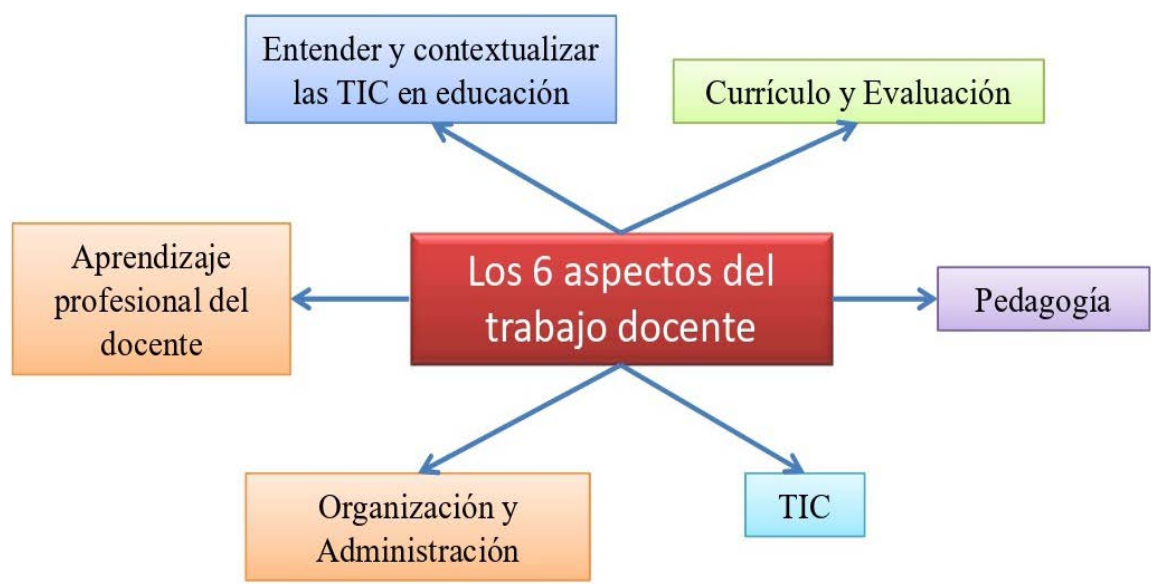

Fuente: UNESCO (2011)

Es necesario que estos aspectos puedan ser desarrollados por los docentes para que contextualicen los recursos TIC en su entorno, adaptando el currículo y la evaluación a partir de los recursos disponibles en el aula y en el centro, a través de una pedagogía activa e innovadora, vinculando a las organizaciones y a la administración.

La última versión del Marco Común de Competencia Digital Docente elaborado por INTEF (2017; omo se cita en Fernández, Fernández y Rodríguez, 2018), muestra las 5 áreas que definen la competencia digital docente, estratificando dicha competencia en 3 niveles de capacitación. Cada uno de estos niveles se subdivide en 2, distinguiendo el grado de capacitación, dentro de ese propio nivel (figura 3). 
Figura 3. Niveles de Competencia Digital Docente

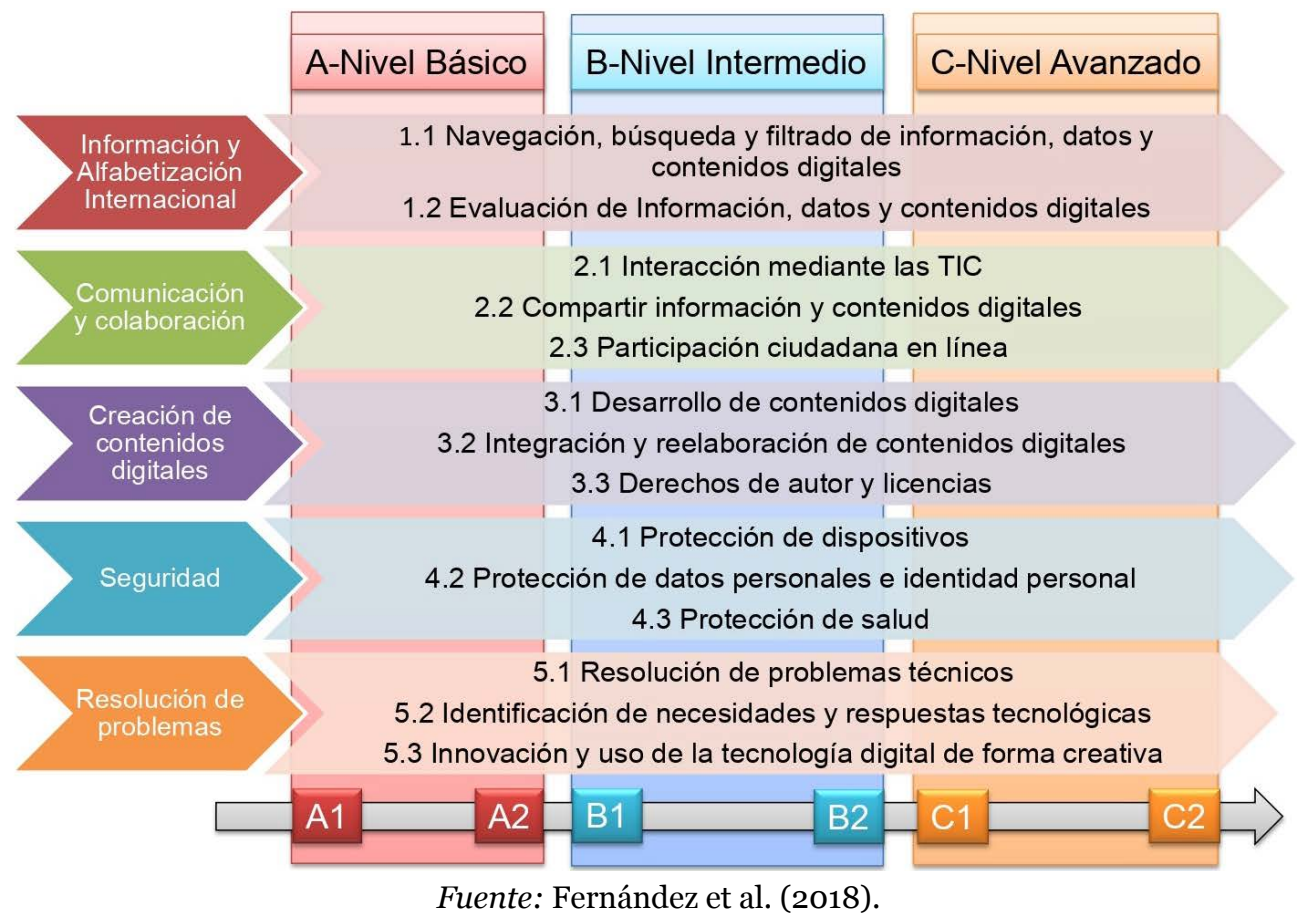

\section{FACTORES DEFINITORIOS DE LA BRECHA DIGITAL}

De acuerdo con Serrano y Martínez (2015; como se cita en Cañón, Grande de Prado y Cantón, 2016), algunos de los principales factores definitorios de la brecha digital, definiéndola como un factor multidimensional (Fernández, 2005) que incorpora determinados factores de acceso, uso y competencia TIC, son: la raza, los ingresos económicos, la localización geográfica, la educación, la edad, el género y la debilidad física.

A nivel internacional, también se han encontrado notables evidencias con respecto a la brecha digital por factores sociales como la edad, la clase social, el género, el nivel educativo o la ubicación geográfica (DiMaggio, Hargittai, Celeste y Shafer, 2004; Livingstone y Helper, 2007; Hargittai y Hinnant, (2008; como se cita en García, Rebollo y García, 2016). Por otra parte, las actitudes y percepciones que poseen los docentes frente a las TIC dependen en gran parte de las variables descriptivas de la brecha digital, como son el género, la edad, el nivel académico y el entorno (Riascos, Ávila y Quintero, 2012). No obstante, los docentes, de forma general, muestran niveles muy bajos de dominio digital, independientemente de la 
edad, la experiencia académica, el nivel de escolaridad y su área de conocimiento (Tapasco y Giraldo, 2017).

Aproximándonos al contexto de nuestra investigación, se observa que los docentes poseen un conjunto de actitudes hacia las TIC más positivas, desarrollando una interacción más efectiva con las tecnologías, permitiéndoles desarrollar una mejor competencia digital docente (Valdés, Arreola, Angulo, Carlos y García, 2011). Con respecto a la edad, diversos estudios y autores indican la existencia de una relación de la competencia digital con la parábola de nativos e inmigrantes digitales. A mayor edad, menor conocimiento y uso de las TIC, y por ello, la predicción de una menor competencia digital (Crovi, 2008; García, Portillo, Romo y Benito, 2007; Lozoya, Cuervo, Armenta, López y Salazar, 2013; Ortega, 2011; Prensky, 2001; 2011). En concreto, a partir de 55 años, la competencia mediática en sus dimensiones más críticas y participativas disminuye de forma significativa con la edad, destacando áreas como la consulta de información y la comunicación. Este rango de edad muestra desconfianza en aplicaciones como el correo electrónico y Facebook (Román y Almansa, 2016).

La experiencia es también considerada como otro valor importante para la definición de la competencia digitaly, además, se encuentra íntimamente relacionada con la edad, ya que el profesorado más novel y con menor experiencia, valora de forma más positiva la potencialidad de los recursos digitales móviles para su uso educativo (Fernández y Fernández, 2016; Vázquez, 2015). La titulación académica del docente es otra variable que no parece tener tanta influencia como las anteriores: no existen grandes diferencias entre los docentes de primaria y secundaria, aunque ambos presentan niveles bajos de competencia digital respecto a los indicadores UNESCO y no se ha observado significatividad en las diferencias de variación entre los grupos con titulaciones de graduado y licenciado (Fernández y Fernández, 2016).

Finalmente, destacamos como factor relevante la localización geográfica, ya que las concentraciones más importantes de conocimientoy, concretamente, las destrezas en el manejo de recursos TIC, se producen en determinadas zonas geográficas, que suelen coincidir con las ciudades más grandes o importantes (capitales o ciudades con mayor población) (UNESCO, 2005; como se cita en Cabero y Ruiz, 2017).

\section{MÉTODO}

\section{Objetivo e hipótesis de estudio}

Con nuestra investigación hemos pretendido dar respuesta al objetivo general: "Determinar las diferencias al acceso a la información, la selección/creación de contenidos y la comunicación como componentes de la competencia digital docente entre los docentes de la provincia de Badajoz". Los objetivos específicos de la investigación son los siguientes: 
1. Observar las diferencias en la capacidad de recoger información en función del género, la edad, los años de experiencia docente, la titulación académica máxima, nivel educativo de docencia y los habitantes del municipio de docencia.

2. Describir las diferencias de selección y creación de contenido en función del género, la edad, los años de experiencia docente, la titulación académica máxima, nivel educativo de docencia y los habitantes del municipio de docencia.

3. Valorar las diferencias en la comunicación docente en función del género, la edad, los años de experiencia docente, la titulación académica máxima, nivel educativo de docencia y los habitantes del municipio de docencia.

A partir de estos objetivos hemos establecido las hipótesis de investigación reflejadas en la figura 4.

Figura 4. Hipótesis de investigación

\section{Género}

H1: Los profesores poseen mejor capacidad de recogida de información que las profesoras.

H7: Los profesores seleccionan y crean contenidos digitales de forma más eficiente que las profesoras.

H13: Los profesores se comunican de forma más eficiente que las profesoras.

\section{Edad}

H2: A medida que aumenta la edad, disminuye la capacidad de recogida de información.

H8: A medida que aumenta la edad, disminuye la capacidad de selección y creación de contenidos.

H14: A medida que aumenta la edad, disminuye la capacidad de comunicación entre los docentes.

\section{Experiencia}

H3: A medida que aumentan los años de experiencia docente disminuye la capacidad de recogida de información.

H9: A medida que aumentan los años de experiencia docente disminuye la capacidad de selección y creación de contenidos.

H15: A medida que aumentan los años de experiencia docente disminuye la comunicación docente.
(Díaz y Cascales, 2015; DiMaggio et al., 2004; Livingstone y Helper, 2007; Hargittai y Hinnant, 2008 citados en García et al., 2016; Dornaleteche, Buitrago y Moreno, 2015; García et al., 2011; Valdés et al., 2011).
(Fernández y Fernández, 2016; Vázquez, 2015). 


\section{Titulación Académica}

\begin{abstract}
H4: A medida que mejora la titulación académica mejora la capacidad de recogida de información.

H10: A medida que mejora la titulación académica mejora la selección y creación de contenidos.

H16: A medida que mejora la titulación académica mejora la comunicación docente.
\end{abstract}

\section{Nivel Educativo}

H5: A medida que aumenta el nivel educativo en el que se imparte clase mejora la capacidad de recogida de información.

H11: A medida que aumenta el nivel educativo en el que se imparte clase aumenta la selección y creación de contenidos.

H17: A medida que aumenta el nivel educativo en el que se imparte clase mejora la comunicación docente.

\section{Situación Geográfica}

H6: A medida que aumentan los habitantes de la población en la que el docente imparte clase mejora la capacidad de recogida de información.

H12: A medida que aumentan los habitantes de la población en la que el docente imparte clase mejora la selección y creación de contenidos.

H18: A medida que aumentan los habitantes de la población en la que el docente imparte clase mejora la comunicación docente.
(Fernández y Fernández, 2016).

(DiMaggio et al., 2004; Livingstone \& Helper, 2007; Hargittai y Hinnant, 2008 citados en García et al., 2016; Fernández y Fernández, 2016).

Fuente: Elaboración propia.

\section{Diseño}

El diseño utilizado para nuestra investigación es cuantitativo, a través de una metodología no experimental, basado en el desarrollo de encuestas a través de un análisis de datos combinado, descriptivo e inferencial (Arnau, 1995; como se cita en Cubo, Luengo, Martín y García, 2011). El diseño de investigación no experimental es aquel en el que no se manipulan las variables, y los datos son obtenidos de la realidad, en este caso, los docentes, y transversal, porque la toma de datos se realiza en un momento único (Hernández, Fernández y Baptista, (2007; como se cita en Romero, Salinas y Mortera, 2010). Varios son los autores que han seguido este modelo de diseño para investigaciones que analizan la competencia digital en docentes y alumnado (Centeno y Cubo, 2013; Fernández, Fernández y Cebreiro, 2016; Martín, Sáenz, Santiago y Chocarro, 2016). 
El desarrollo de la investigación se ha llevado a cabo en cuatro fases, elaboradas a partir del diseño de planificación del Proyecto de Investigación realizado por Inche, Andía, Huamanchumo, López, Vizcarra y Flores (2003), ejemplificado en la figura 5.

Figura 5. Planificación del Proyecto de Investigación

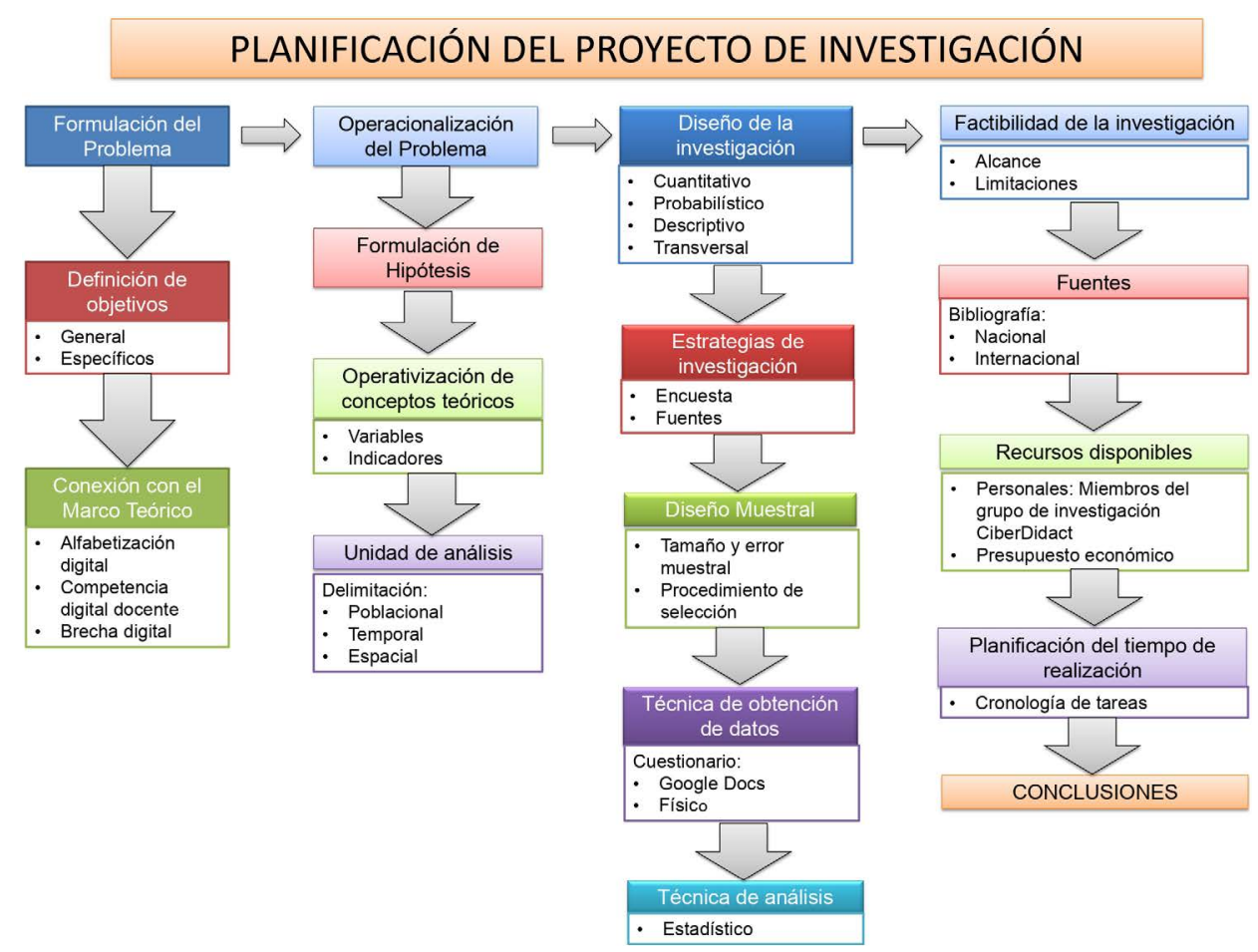

Fuente: a partir de D’Áncora (1996; como se cita en Inche et al., 2003).

1. Formulación del problema: Se realiza una primera toma de contacto con el problema de investigación, definiendo el objetivo general de investigación y los específicos, para posteriormente contextualizarlos a través del marco teórico.

2. Operacionalización del problema: Se profundiza en el planteamiento del problema de investigación, formulando las hipótesis de trabajo, operativizando los conceptos teóricos como son las variables que utilizamos en nuestra investigación, y los indicadores de calidad y validez. Posteriormente, fijamos las unidades de análisis, estableciendo las delimitaciones poblacionales, temporales y espaciales de la investigación.

3. Diseño de investigación: Se contextualiza el diseño de investigación a las características del planteamiento del problema, desarrollando las estrategias de investigación, a través de la encuesta y de las fuentes observadas. A continuación, 
se realiza el diseño muestral, analizando el tamaño y el error muestral, y valorando el procedimiento de selección. Se procede a la elección de la técnica de obtención de datos a través de cuestionario, facilitándolo a la muestra en formato físico a las poblaciones menores de 10.000 habitantes y a través de google drive a las poblaciones con más de 10.000 habitantes. A continuación, se establece la técnica de obtención de datos, a través de un análisis estadístico.

4. Finalmente, analizamos la factibilidad de la investigación, observando su alcance y limitaciones, para posteriormente, correlacionar los resultados con las fuentes bibliográficas nacionales e internacionales consultadas. Observamos los recursos disponibles para el desarrollo de la investigación, tanto los personales, formados por los miembros del grupo de investigación CiberDidact de la Facultad de Educación de Badajoz, como los económicos. Se establece la planificación del tiempo de realización a través de un marco cronológico de las tareas a realizar. En último lugar, se establecerán las conclusiones a partir del marco teórico y de los datos obtenidos.

\section{Muestra}

Se ha trabajado con una estimación de población obtenida a través del Instituto de Estadística de Extremadura ${ }^{1}$, que nos arrojaba las cifras de docentes en función de los niveles educativos que reflejamos en la tabla 1.

Tabla 1. Estimación de la Población

\begin{tabular}{|c|c|}
\hline Nivel educativo & Total de docentes \\
\hline Infantil y Primaria & 4906 \\
\hline Secundaria, Bachillerato & 4397 \\
\hline Formación de adultos & 316 \\
\hline Universidad de Extremadura & 1851 \\
\hline Total & 11470 \\
\hline
\end{tabular}

Fuente: Elaboración propia.

De igual modo, se ha trabajado con un nivel de confianza del 95\% y un error muestral del $5 \%$ a través de la fórmula del cálculo de tamaño de población (Vallejo, 2012); el resultado obtenido fue de 372 docentes. Se optó por seleccionar un total de 400 docentes de los 434 docentes que fueron encuestados para obtener una mejor estimación.

En la tabla 2 reflejamos las características de la muestra. 
Tabla 2. Características de la muestra

\begin{tabular}{|c|c|c|}
\hline \multicolumn{3}{|c|}{ Género } \\
\hline Variable & Frecuencia & Porcentaje \\
\hline Hombre & 167 & $41,8 \%$ \\
\hline Mujer & 233 & $58,2 \%$ \\
\hline \multicolumn{3}{|c|}{ Edad } \\
\hline Menos de 31 años & 23 & $5,8 \%$ \\
\hline Entre 31 y 40 años & 105 & $26,3 \%$ \\
\hline Entre 41 y 50 años & 143 & $35,6 \%$ \\
\hline Entre 51 y 60 años & 117 & $29,3 \%$ \\
\hline Más de 60 años & 13 & $3 \%$ \\
\hline \multicolumn{3}{|c|}{ Años de experiencia del profesorado } \\
\hline Menos de 11 & 117 & $29,3 \%$ \\
\hline Entre 11 y 20 años & 133 & $33,3 \%$ \\
\hline Entre 21 y 31 años & 93 & $23,3 \%$ \\
\hline Más de 31 años & 57 & $14,1 \%$ \\
\hline \multicolumn{3}{|c|}{ Titulación académica máxima } \\
\hline Diplomatura o grado & 148 & $37 \%$ \\
\hline Licenciatura & 150 & $37,5 \%$ \\
\hline Máster & 74 & $18,5 \%$ \\
\hline Doctor & 28 & $7 \%$ \\
\hline \multicolumn{3}{|c|}{ Nivel educativo de docencia } \\
\hline Infantil o primaria & 161 & $40,3 \%$ \\
\hline $\begin{array}{l}\text { Educación secundaria, Bachillerato } \\
\text { o formación profesional }\end{array}$ & 117 & $29,3 \%$ \\
\hline Universidad & 122 & $30,4 \%$ \\
\hline \multicolumn{3}{|c|}{ Habitantes del municipio de docencia } \\
\hline Menos de 10.000 habitantes & 99 & $24,8 \%$ \\
\hline Entre 10.001 y 40.000 habitantes & 71 & $17,8 \%$ \\
\hline Entre 40.001 y 70.000 habitantes & 37 & $9,3 \%$ \\
\hline Más de 70.00o habitantes & 193 & $48,3 \%$ \\
\hline
\end{tabular}

Fuente: Elaboración propia.

\section{Instrumento}

Para el desarrollo de nuestra investigación hemos utilizado el instrumento para la evaluación de la competencia digital docente, desarrollado por Martín et al. (2016), del que hemos seleccionado tres de las cinco dimensiones que lo componen (Búsqueda de información, Selección/creación de contenidos y Comunicación). 
Hemos realizado pequeñas modificaciones en la dimensión de datos personales y en alguno de los ítems, para ajustar el instrumento a los objetivos de nuestra investigación. Este instrumento ha servido de base para la creación de otros instrumentos para medir la competencia digital como el Estudio Psicométrico de un Cuestionario para medir la Competencia Digital en Estudiantes Universitarios (CODIEU) (Cabezas, Casillas, Chánches y Teixeira, 2018). En la tabla 3 reflejamos las dimensiones utilizadas y su fiabilidad. Cada ítem de escala del cuestionario se compone de dos variables: el conocimiento que el docente considera que posee sobre el ítem analizado y el uso que el ítem tiene para el docente en el aula.

Tabla 3. Características y fiabilidad de las dimensiones y del instrumento

\begin{tabular}{|l|c|c|}
\hline \multicolumn{1}{|c|}{ Dimensión } & $\begin{array}{c}\text { No de } \\
\text { ítems }\end{array}$ & $\begin{array}{c}\text { Fiabilidad (Alfa } \\
\text { de Cronbach) }\end{array}$ \\
\hline $\begin{array}{l}\text { Búsqueda de información (Identificar, localizar, recuperar, } \\
\text { almacenar, organizar y analizar la información digital, } \\
\text { evaluando su finalidad y relevancia). }\end{array}$ & 16 & 0,935 \\
\hline $\begin{array}{l}\text { Selección/creación de contenidos (Crear y editar contenidos } \\
\text { nuevos -textos, imágenes, videos...-, integrar y reelaborar } \\
\text { conocimientos y contenidos previos, realizar producciones } \\
\text { artísticas, contenidos multimedia y programación informática, } \\
\text { saber aplicar los derechos de propiedad intelectual y las } \\
\text { licencias de uso). }\end{array}$ & 24 & 0,938 \\
\hline $\begin{array}{l}\text { Comunicación (Comunicar en entornos digitales, compartir } \\
\text { recursos a través de herramientas en línea, conectar y } \\
\text { colaborar con otros a través de herramientas digitales, } \\
\text { interactuar y participar en comunidades y redes; conciencia } \\
\text { intercultural). }\end{array}$ & 14 & 0,921 \\
\hline Cuestionario completo & 56 & 0,966 \\
\hline
\end{tabular}

Fuente: a partir de Martín et al. (2016).

\section{RESULTADOS}

\section{Análisis descriptivo}

En el análisis descriptivo realizado hemos analizado de forma global cada una de las dimensiones objeto de estudio, en sus dos variables (conocimiento y uso). En la figura 6 se disponen los resultados de la dimensión "Búsqueda de información". 
Figura 6. Promedios de los ítems de la dimensión 1 ("Búsqueda de información”)

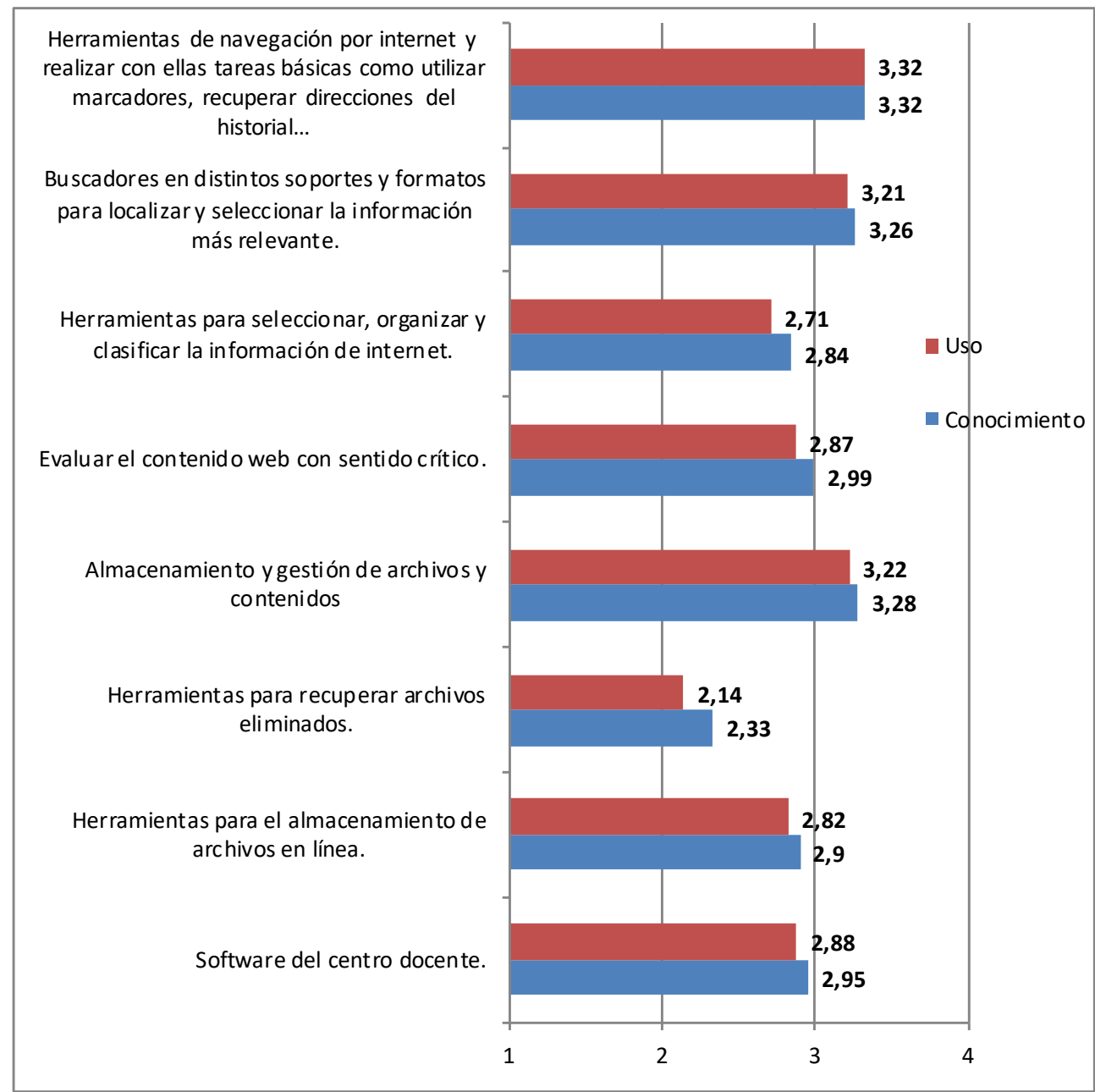

Fuente: Elaboración propia.

En la dimensión 1, "Búsqueda de información", los docentes destacan el conocimiento en "Herramientas de navegación por internet y realizar con ellas tareas básicas como utilizar marcadores, recuperar direcciones del historial..." (M $=3,32 ; \mathrm{DT}=0,836) \mathrm{y}$ "Almacenamiento y gestión de archivos y contenidos" $(\mathrm{M}=$ 3,28; DT $=0,873$ ). Por otra, los conocimientos en búsqueda de información que los docentes consideran menos importantes son las "Herramientas para recuperar 
archivos eliminados" $(\mathrm{M}=2,33$; DT = 1,080 $)$ y las "Herramientas para seleccionar, organizar y clasificar la información...” ( $\mathrm{M}=2,84$; $\mathrm{DT}=1,029)$.

Para la variable de uso de los ítems de "Búsqueda de información", los docentes destacan las "Herramientas de navegación por internet y realizar con ellas tareas básicas como utilizar marcadores, recuperar direcciones del historial..." $(\mathrm{M}=3,32$; $\mathrm{DT}=0,903)$ y el "Almacenamiento y gestión de archivos y contenidos" $(\mathrm{M}=3,22$; DT $=0,922$ ). No obstante, los recursos de búsqueda de información menos usados por los docentes son las "Herramientas para recuperar archivos eliminados" (M $=2,14$; DT $=1,013$ ) y las "Herramientas para seleccionar, organizar y clasificar la información...” (M = 2,71; DT = 1,057).

Podemos observar cómo de forma general se obtienen mayores puntuaciones medias para los valores de conocimiento del ítem que de uso del ítem, salvo para el ítem "Herramientas de navegación por Internet y realizar con ellas tareas básicas como utilizar marcadores, recuperar direcciones del historial..." en el que ambos tienen la misma puntuación media. Los docentes valoran ligeramente en mayor medida el conocimiento de la búsqueda de información $(\mathrm{M}=2,98$; $\mathrm{DT}=0,723)$ que el uso de la búsqueda de información ( $\mathrm{M}=2,88 ; \mathrm{DT}=0,716)$.

En la figura 7 se disponen los resultados de la dimensión "Selección/creación de contenido". 
Figura 7. Promedios de los ítems de la dimensión 2 (“Selección/creación de contenidos”)

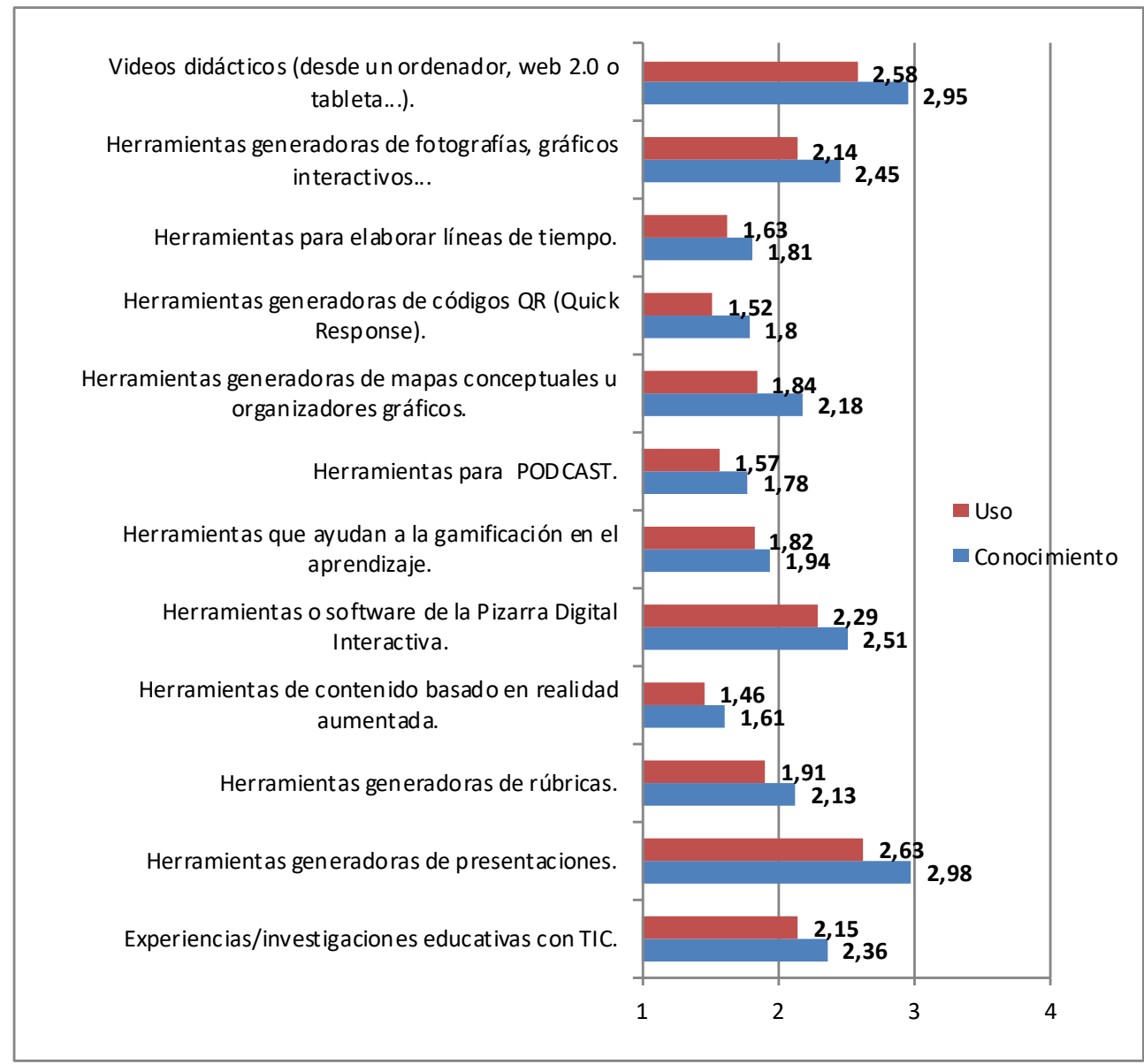

Fuente: Elaboración propia.

En la dimensión 2, "Selección y creación de contenidos", los docentes destacan el conocimiento en "Herramientas generadoras de presentaciones" $(\mathrm{M}=2,98$; DT = 1,040) y "Videos didácticos (desde un ordenador, web 2.0 o tableta...)" (M= 2,95; DT = 1,079). En cambio, los docentes poseen menos conocimiento de "Herramientas de contenido basadas en realidad aumentada" $(\mathrm{M}=1,61 ; \mathrm{DT}=0,965)$ y de "Herramientas para PODCAST" $(\mathrm{M}=1,78 ; \mathrm{DT}=1,068)$.

Para la variable de uso del ítem de "Selección/creación de contenidos", los docentes destacan el uso de "Herramientas generadoras de presentaciones" ( $\mathrm{M}=$ 2,63; DT = 1,018) y de "Videos didácticos (desde un ordenador, web 2.0 o tableta,...)" 
( $\mathrm{M}=2,58 ; \mathrm{DT}=1,057)$ coincidiendo con las puntuaciones medias más elevadas de conocimiento. No obstante, los recursos de selección y creación de contenidos menos usados por los docentes son las "Herramientas de contenido basadas en realidad aumentada" ( $\mathrm{M}=1,46 ; \mathrm{DT}=0,846)$ y las "Herramientas generadoras de códigos QR (Quick Response)" (M = 1,52; DT = 0,870).

Podemos observar cómo de forma general se obtienen mayores puntuaciones medias para los valores de conocimiento del ítem que de uso del ítem. Los docentes valoran de forma más positiva el conocimiento de la selección/creación de contenidos $(\mathrm{M}=2,21 ; \mathrm{DT}=0,748)$ que el uso de la selección/creación de contenidos $(\mathrm{M}=1,96$; $\mathrm{DT}=0,665)$.

En la figura 8 se disponen los resultados de la dimensión "Comunicación".

Figura 8. Promedios de los ítems de la dimensión 3 (“Comunicación”)

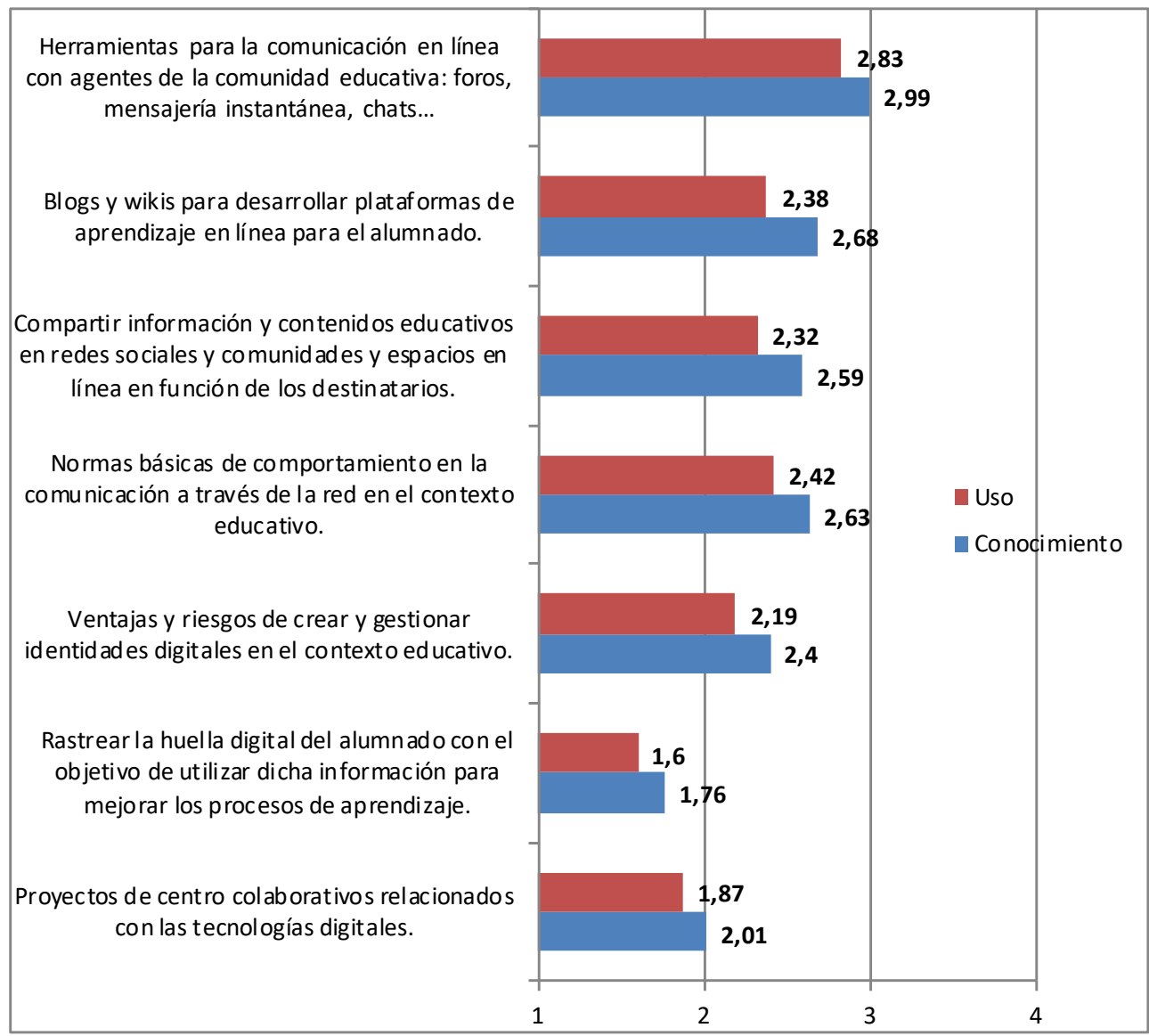

Fuente: Elaboración propia. 
En la dimensión 3, "Comunicación", los docentes destacan el conocimiento en "Herramientas para la comunicación en línea con agentes de la comunidad educativa: foros, mensajería..." ( $\mathrm{M}=2,99$; $\mathrm{DT}=1,045)$ y en "Blog y wikis para desarrollar plataformas de aprendizaje en línea para el alumnado" $(\mathrm{M}=2,68$; DT = 1,130). En cambio, los docentes tienen menos conocimiento en "Rastrear la huella digital del alumnado con el objetivo de utilizar dicha información para mejorar los procesos de aprendizaje" ( $\mathrm{M}=1,76$; DT 1,053) y "Proyectos de centro colaborativos relacionados con las tecnologías digitales" $(\mathrm{M}=2,01 ; 1,096)$.

Para la variable de uso del ítem de "Comunicación", los docentes destacan el uso de "Herramientas para la comunicación en línea con agentes de la comunidad educativa: foros, mensajería..." ( $\mathrm{M}=2,83$; DT $=1,050)$ y de "Normas básicas de comportamiento en la comunicación a través de la red en el contexto educativo" (M $=2,42 ; \mathrm{DT}=1,123$ ). No obstante, los recursos menos utilizados para la comunicación por los docentes son "Rastrear la huella digital del alumnado con el objetivo de utilizar dicha información para mejorar los procesos de aprendizaje" (D = 1,06; $\mathrm{DT}=0,912)$ y "Proyectos de centro colaborativos relacionados con las tecnologías digitales" $(\mathrm{M}=1,87$; $\mathrm{DT}=1,024)$.

Podemos observar cómo de forma general se obtienen mayores puntuaciones medias para los valores de conocimiento del ítem que de uso del ítem. Los docentes valoran de forma ligeramente más positiva el conocimiento en comunicación ( $\mathrm{M}=$ 2,44; DT = 0,823) que el uso de la comunicación $(\mathrm{M}=2,23 \mathrm{DT}=0,753)$.

Finalmente, analizamos las puntuaciones medias de cada una de las dimensiones en función del nivel mínimo recomendado. Como podemos observar en la figura 9, solamente la dimensión 1: "Búsqueda de información", se encuentra por encima del nivel mínimo recomendado $(\mathrm{M}=2,93 ; \mathrm{DT}=0,707)$, mientras que las dimensiones 2 y 3: "Selección y creación de contenidos" y "Comunicación" $(\mathrm{M}=2,08 ; \mathrm{DT}=0,674)$ y $(\mathrm{M}=2,33 ; \mathrm{DT}=0,762)$, respectivamente, se encuentran por debajo del nivel mínimo recomendado.

Figura 9. Promedios de las dimensiones analizadas

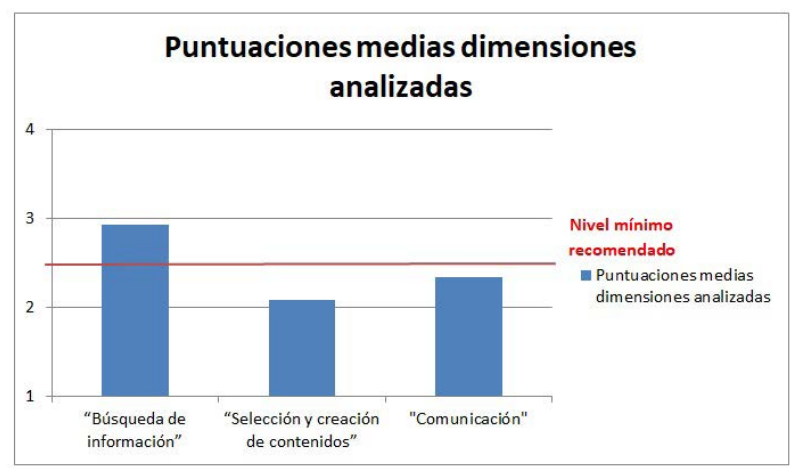

Fuente: Elaboración propia. 


\section{Análisis inferencial}

Una vez realizado el análisis descriptivo preliminar, se procedió a realizar el análisis inferencial mediante el uso del programa estadístico SPSS en su versión 21 trabajando con un nivel de significación p<0,05. En primer lugar, se empleó la prueba de Kolmogorov-Smirnov para observar si la muestra se adaptaba a la curvatura normal; posteriormente, se ejecutó la prueba de Rachas, para confirmar la aleatorización de la muestra. Finalmente, se ejecutó la prueba de Levene, en los casos requeridos, para observar la homocedasticidad de la muestra. Con los datos obtenidos se seleccionó el tipo de prueba a utilizar (paramétrica o no paramétrica) en función de los resultados observados y el conjunto de grupos a analizar. Reflejamos los resultados en la tabla 4.

Tabla 4. Resultados pruebas Kolmogorov-Smirnov y Rachas

\begin{tabular}{|c|c|c|c|}
\hline Variable & K-S & Rachas & Prueba a realizar \\
\hline Información & $\mathrm{p}=0,000$ & $\mathrm{p}=0,110$ & No paramétrica \\
\hline $\begin{array}{c}\text { Selección y creación de } \\
\text { contenidos }\end{array}$ & $\mathrm{p}=0,000$ & $\mathrm{p}=0,480$ & No paramétrica \\
\hline Comunicación & $\mathrm{p}=0,007$ & $\mathrm{p}=0,000$ & No paramétrica \\
\hline
\end{tabular}

Fuente: Elaboración propia.

Los resultados indican que existen diferencias significativas en la dimensión de información, en función del género, la edad, la titulación del docente y los habitantes del municipio (tabla 5). Por ello, se rechaza la hipótesis nula y se acepta la hipótesis de trabajo para los casos:

- "Los profesores poseen mejor capacidad de recogida de información que las profesoras" $(\mathrm{p}=0,002)$.

- "A medida que aumenta la edad, disminuye la capacidad de recogida de información" $(\mathrm{p}=0,035)$.

- "A medida que mejora la titulación académica mejora la capacidad de recogida de información" ( $\mathrm{p}=0,000)$.

- "A medida que aumentan los habitantes de la población en la que el docente imparte clase mejora la capacidad de recogida de información" ( $p=0,001)$. 
Tabla 5. Resultados de las hipótesis de la dimensión 1 ("Búsqueda de información”)

\begin{tabular}{|c|c|c|}
\hline Hipótesis & Prueba & Implicaciones \\
\hline 1 & $\begin{array}{l}\text { Modelo no paramétrico: U de Mann } \\
\text { Whitney }\end{array}$ & $\begin{array}{c}\mathrm{p}=0,002(\text { Se rechaza la Ho y se acepta } \\
\left.\text { la } \mathrm{H}_{1}\right)\end{array}$ \\
\hline 2 & $\begin{array}{l}\text { Modelo no paramétrico: Prueba } \mathrm{H} \text { de } \\
\text { Kruskal Wallis }\end{array}$ & $\begin{array}{c}\mathrm{p}=0,035(\text { Se rechaza la Ho y se acepta } \\
\left.\text { la } \mathrm{H}_{1}\right)\end{array}$ \\
\hline 3 & $\begin{array}{l}\text { Modelo no paramétrico: Prueba } \mathrm{H} \text { de } \\
\text { Kruskal Wallis }\end{array}$ & $\begin{array}{c}\mathrm{p}=0,162(\text { Se acepta la Ho y se rechaza } \\
\left.\text { la } H_{1}\right)\end{array}$ \\
\hline 4 & $\begin{array}{l}\text { Modelo no paramétrico: Prueba } \mathrm{H} \text { de } \\
\text { Kruskal Wallis }\end{array}$ & $\begin{array}{c}\mathrm{p}=0,000(\text { Se rechaza la Ho y se acepta } \\
\left.\text { la } \mathrm{H}_{1}\right)\end{array}$ \\
\hline 5 & $\begin{array}{l}\text { Modelo no paramétrico: Prueba } \mathrm{H} \text { de } \\
\text { Kruskal Wallis }\end{array}$ & $\begin{array}{c}\mathrm{p}=0,078(\text { Se rechaza la Ho y se acepta } \\
\left.\text { la } \mathrm{H}_{1}\right)\end{array}$ \\
\hline 6 & $\begin{array}{l}\text { Modelo no paramétrico: Prueba } \mathrm{H} \text { de } \\
\text { Kruskal Wallis }\end{array}$ & $\begin{array}{c}\mathrm{p}=0,001(\text { Se rechaza la Ho y se acepta } \\
\left.\text { la } \mathrm{H}_{1}\right)\end{array}$ \\
\hline
\end{tabular}

Fuente: Elaboración propia.

Los resultados indican que existen diferencias significativas en la dimensión de "Selección y creación de contenidos" en función de la edad, la experiencia docente, la titulación máxima, en nivel de docencia y el municipio (tabla 6):

- “A medida que aumenta la edad, disminuye la capacidad de selección y creación de contenidos" ( $\mathrm{p}=\mathrm{o}, \mathrm{ooo})$.

- "A medida que aumentan los años de experiencia docente disminuye la capacidad de selección y creación de contenidos" $(\mathrm{p}=\mathrm{0}, \mathrm{O} 11)$.

- "A medida que mejora la titulación académica mejora la selección y creación de contenidos" ( $\mathrm{p}=0,035)$.

- "A medida que aumenta el nivel educativo en el que se imparte clase mejora la selección y creación de contenidos” $(\mathrm{p}=0,012)$.

- "A medida que aumentan los habitantes de la población en la que el docente imparte clase mejora la selección y creación de contenidos" ( $p=0,014)$.

Tabla 6. Resultados de las hipótesis de la dimensión 2 ("Selección/creación de contenidos”)

\begin{tabular}{|c|c|c|}
\hline Hipótesis & Prueba & Implicaciones \\
\hline 7 & $\begin{array}{c}\text { Modelo no paramétrico: U de Mann } \\
\text { Whitney }\end{array}$ & $\begin{array}{c}\mathrm{p}=0,413(\text { Se acepta la Ho y se rechaza } \\
\left.\text { la } \mathrm{H}_{1}\right)\end{array}$ \\
\hline 8 & $\begin{array}{l}\text { Modelo no paramétrico: Prueba } \mathrm{H} \text { de } \\
\text { Kruskal Wallis }\end{array}$ & $\begin{array}{c}\mathrm{p}=0, \mathrm{ooo}(\text { Se rechaza la Ho y se acepta } \\
\left.\text { la } \mathrm{H}_{1}\right)\end{array}$ \\
\hline 9 & $\begin{array}{c}\text { Modelo no paramétrico: Prueba } \mathrm{H} \text { de } \\
\text { Kruskal Wallis }\end{array}$ & $\begin{array}{c}\mathrm{p}=0,011(\text { Se rechaza la Ho y se acepta } \\
\left.\text { la } \mathrm{H}_{1}\right)\end{array}$ \\
\hline
\end{tabular}




\begin{tabular}{|c|c|c|}
\hline Hipótesis & Prueba & Implicaciones \\
\hline 10 & $\begin{array}{l}\text { Modelo no paramétrico: Prueba H de } \\
\text { Kruskal Wallis }\end{array}$ & $\begin{array}{c}\mathrm{p}=0,035(\text { Se rechaza la Ho y se acepta } \\
\left.\text { la } \mathrm{H}_{1}\right)\end{array}$ \\
\hline 11 & $\begin{array}{l}\text { Modelo no paramétrico: Prueba H de } \\
\text { Kruskal Wallis }\end{array}$ & $\begin{array}{c}\mathrm{p}=0,012(\text { Se rechaza la Ho y se acepta } \\
\left.\text { la } \mathrm{H}_{1}\right)\end{array}$ \\
\hline 12 & $\begin{array}{l}\text { Modelo paramétrico: Anova de un } \\
\text { factor }\end{array}$ & $\begin{array}{c}\mathrm{p}=0,014(\text { Se rechaza la Ho y se acepta } \\
\left.\text { la } \mathrm{H}_{1}\right)\end{array}$ \\
\hline
\end{tabular}

Fuente: Elaboración propia.

Los resultados indican que existen diferencias significativas en la dimensión de "Comunicación" en función de la edad, la experiencia docente y la titulación máxima (tabla 7):

- "A medida que aumenta la edad, disminuye la capacidad de comunicación entre los docentes" $(\mathrm{p}=0,007)$.

- "A medida que aumentan los años de experiencia docente disminuye la comunicación docente" $(0,019)$.

- "A medida que mejora la titulación académica mejora la comunicación docente" $(0,000)$.

Tabla 7. Resultados de las hipótesis de la dimensión 3 (“Comunicación”)

\begin{tabular}{|c|c|c|}
\hline Hipótesis & Prueba & Implicaciones \\
\hline 13 & $\begin{array}{l}\text { Modelo no paramétrico: U de Mann } \\
\text { Whitney }\end{array}$ & $\begin{array}{c}\mathrm{p}=0,240 \text { (Se acepta la Ho y se rechaza } \\
\left.\text { la } \mathrm{H}_{1}\right)\end{array}$ \\
\hline 14 & $\begin{array}{l}\text { Modelo no paramétrico: Prueba H de } \\
\text { Kruskal Wallis }\end{array}$ & $\begin{array}{c}\mathrm{p}=0,007(\text { Se rechaza la Ho y se acepta } \\
\left.\text { la } \mathrm{H}_{1}\right)\end{array}$ \\
\hline 15 & $\begin{array}{l}\text { Modelo no paramétrico: Prueba H de } \\
\text { Kruskal Wallis }\end{array}$ & $\begin{array}{c}\mathrm{p}=0,019(\text { Se rechaza la Ho y se acepta } \\
\left.\text { la } \mathrm{H}_{1}\right)\end{array}$ \\
\hline 16 & $\begin{array}{l}\text { Modelo no paramétrico: Prueba } \mathrm{H} \text { de } \\
\text { Kruskal Wallis }\end{array}$ & $\begin{array}{c}\mathrm{p}=0,000(\text { Se rechaza la Ho y se acepta } \\
\left.\text { la } \mathrm{H}_{1}\right)\end{array}$ \\
\hline 17 & $\begin{array}{l}\text { Modelo no paramétrico: Prueba } \mathrm{H} \text { de } \\
\text { Kruskal Wallis }\end{array}$ & $\begin{array}{c}\mathrm{p}=0,107(\text { Se acepta la Ho y se rechaza } \\
\left.\text { la } \mathrm{H}_{1}\right)\end{array}$ \\
\hline 18 & $\begin{array}{c}\text { Modelo no paramétrico: Prueba } \mathrm{H} \text { de } \\
\text { Kruskal Wallis }\end{array}$ & $\begin{array}{c}\mathrm{p}=0,067(\text { Se acepta la Ho y se rechaza } \\
\left.\text { la } \mathrm{H}_{1}\right)\end{array}$ \\
\hline
\end{tabular}

Fuente: Elaboración propia. 


\section{DISCUSIÓN Y CONCLUSIONES}

A tenor de los resultados obtenidos, coincidimos con Durán, Gutiérrez y Prendes (2016, p. 112) en que "las dimensiones que definen la competencia digital, tecnológica, comunicativa, informacional, multimedia, de seguridad y resolución de problemas, no solo deben ser observadas como competencias presentes en los entornos digitales, sino para ayudar a la mejora de la ciudadanía y formación", y se deben mostrar como requisitos básicos para poder formar al alumnado.

Coincidimos con Pérez y Rodríguez (2016), quienes nos ponen de manifiesto la necesidad de reforzar los modelos que fomentan el desarrollo de la competecia digital docente desde diferentes ámbitos, como los referidos al ámbito educativo, tanto a nivel internacional, como la metodología TPACK (Shulman, 1986; Mishra y Kolher, 2006, 2008), el modelo noruego (Krumsvik, 2008, 2011), el ISTE Standards para profesores, NETS-T (ISTE, 2008) desarrollado en Estados Unidos o documentos Europeos como el Proyecto ECD-TIC (UNESCO, 2008) o el Currículum AMI (UNESCO, 2011); pero también a nivel nacional, como los documentos INTEF (2017), Consejería de Educación de Castilla y León, Red XXI Educacyldigital (2010). Coincidimos, por tanto, en que resulta imprescindible para el profesorado poder dominar las dimensiones que componen la competencia digital, para poderlas mostrar y enseñar al alumnado (González, Espuny, de Cid y Gisbert, 2012). Por ello, coincidimos en que resulta indispensable la investigación para poder mejorar la capacitación digital docente en los diferentes entornos, relacionando las variables que pueden ser significativas para su mejora.

En el análisis de las tres dimensiones de estudio (Búsqueda de información, Selección y creación de contenidos y Comunicación) se ha observado que los docentes dotan de mayor importancia a la búsqueda de información como componente de la competencia digital (Arroyo, 2017; Domingo y Marqués, 2011), siendo un elemento indispensable para la docencia en la sociedad del conocimiento, permitiendo la elaboración, difusión y acceso a la información (Area y Guarro, 2012) mediante el uso de herramientas de navegación por internet, constituyendo la búsqueda de información a través de la Web una de las actividades más básicas, pero a la vez una de las fundamentales para el alumnado (San Nicolás, Fariña y Area, 2012).

Dando respuesta a los objetivos de investigación, los resultados muestran que en función del género, se encuentran diferencias en la capacidad y el conocimiento del acceso a la información. La edad y el nivel máximo de formación del docente resultan determinantes, ya que influyen en las tres dimensiones: Búsqueda de información, Selección y creación de contenidos y Comunicación. En la experiencia docente se muestran diferencias significativas en la selección y creación de contenidos, de igual modo que en la comunicación. Dependiendo del nivel en el que imparte la enseñanza el docente, se observan diferencias significativas únicamente en la selección y creación de contenidos. Finalmente, el tipo de municipio en función de su población muestra diferencias significativas en la capacidad para obtener información y en la selección 
y creación de contenidos. Son por tanto constatables las diferencias significativas existentes en factores que definen la competencia digital, en variables influyentes de la brecha digital (Serrano y Martínez, 2015; como se cita en Cañón et al., 2016).

Consideramos, por tanto, el importantísimo papel que toma la competencia digital para el profesorado y el alumnado, confirmándose que, a medida que se tiene un mayor conocimiento de las herramientas y los recursos TIC, en competencias como la información, la selección y creación de contenidos y la comunicación, mejorará la competencia digital (Centeno y Cubo, 2013; Cabanillas, Luengo y Carvalho, 2018, 2019) y, de forma general, la competencia digital ha sido incorporada en el conjunto de componentes que forman la competencia docente del profesorado en cualquier nivel educativo.

\section{NOTAS}

1. Instituto de Estadística de Extremadura (https://ciudadano.gobex.es/web/ieex)

\section{REFERENCIAS}

Area, M., y Guarro, A. (2012). La alfabetización informacional y digital: fundamentos pedagógicos para la enseñanza y el aprendizaje competente. Revista española de documentación científica, 35(Monográfico), 46-74. doi: https://doi.org/10.3989/redc.2012. mono.977

Arnau, J. (1995). Conceptos básicos. En M. T. Angera, J. Arnau, M. Ato, R. Martínez, J. Pascual y G. Vallejo (Eds.), Métodos de investigación en psicología (45-72). Madrid: Síntesis.

Arroyo, A. (2017). Docentes y escuelas que aprenden en la red. Estudio sobre la competencia digital, entornos personales de aprendizaje y entorno organizacional de aprendizaje de Arizmendi ikastola (tesis doctoral). Madrid: UNED. Recuperado de http://e-spacio.uned. es/fez/eserv/tesisuned:ED-Pg-CyEEDAarroyo/ARROYO SAGASTA Amaia Tesis.pdf

Cabanillas, J. L., Luengo, R., y Carvalho, J. L. T. (2018). Correlación entre el conocimiento, actitud hacia las TIC y las emociones en el máster universitario de formación del profesorado y TIC. Revista Internacional de Tecnologías en la Educación, 5(2), 69-79. Recuperado de https://journals.epistemopolis.org/ index.php/tecnologiasedu/article/ view/1820/1194

Cabanillas, J. L., Luengo, R., y Carvalho, J. L. T. (2019). Diferencias de actitud hacia las TIC en la formación profesional en entornos presenciales y virtuales. PixelBit, Revista de Medios y Educación, 55, 37-55. doi: https://doi.org/10.12795/ pixelbit.2019.i55.03

Cabero, J., y Ruiz, J. (2017). Las Tecnologías de la Información y Comunicación para la inclusión: reformulando la brecha digital. Ijeri. International Journal of Educational Research and Innovation, 9, 16-30. Recuperado de https://idus.us.es/xmlui/bitstream/ handle/11441/66918/2665-8692-1-PB. pdf?sequence $=1$ \&isAllowed $=\mathrm{y}$

Cabezas, M., Casillas, S., y Pinto, A. M. (2014). Percepción de los alumnos de Educación Primaria de la universidad 
de Salamanca sobre su competencia digital. EDUTEC, Revista Electrónica de Tecnología Educativa, 48. doi: http:// dx.doi.org/10.21556/edutec.2014.48.156

Cañón, R., Grande de Prado, M., y Cantón, I. (2016). Brecha digital: impacto en el desarrollo social y personal. Factores asociados. Tendencias pedagógicas, (28), 115-132. Recuperado de https:// repositorio.uam.es/bitstream/ handle/10486/671836/TP 2810. pdf?sequence $=1$

Centeno, G., y Cubo, S. (2013). Evaluación de la competencia digital y las actitudes hacia las TIC del alumnado universitario. Revista de Investigación Educativa, 31(2), 517-536. Recuperado de http://revistas.um.es/rie/article/ download/169271/158221

Crovi, D. (2008). Diagnóstico acerca del acceso, uso y apropiación de las TIC en la UNAM. Anuario ININCO, 2O(1), 79-96. Recuperado de http://saber. ucv.ve/ojs/index.php/rev ai/article/ download/5282/5082

Cubo, S., Luengo, R., Martín, B., y García, J. L. (Coord.) (2011). Métodos de investigación $y$ análisis de datos en ciencias sociales $y$ de la salud. Madrid: Ediciones Pirámide.

D’Áncora, M. A. (1996). Metodología Cuantitativa: Estrategias y Técnicas de Investigación Social. Madrid: Editorial Síntesis.

Díaz, I. T., y Casacales, A. (2015). Las TIC $\mathrm{y}$ las necesidades específicas de apoyo educativo: análisis de las competencias TIC en los docentes. RIED. Revista Iberoamericana de Educación a Distancia, 18(2), 355-383. Recuperado de https://www.redalyc.org/ pdf/3314/331439257015.pdf

DiMaggio, P., Hargittai, E., Celeste, C., y Shafer, S. (2004). Digital inequality: From unequal access to differentiated use. En K. Neckerman (Ed.), Social Inequality (355400). New York: Russell Sage Foundation.
Domingo, M., y Marquès, P. (2011). Aulas 2.o y uso de las TIC en la práctica docente. Comunicar, 19(37), 169-175. doi: http:// dx.doi.org/10.3916/C37-2011-03-09

Dornaleteche, J., Buitrago, A., y Moreno, L. (2015). Categorización, selección de ítems y aplicación del test de alfabetización digital online como indicador de la competencia mediática. Comunicar, 22(44), 177-185. doi: http://dx.doi. org/10.3916/C44-2015-19

Durán, M., Gutiérrez, I., y Prendes, M. P. (2016). Certificación de la competencia TIC del profesorado universitario: Diseño y validación de un instrumento. Revista mexicana de investigación educativa, 21(69), 527-556. Recuperado de http://www.scielo.org.mx/scielo.php?script =sci arttext\&pi$\mathrm{d}=\mathrm{S} 1405-66662016000200527$

Fernández, F. J. (2005). Brecha e inclusión digital en Chile: los desafíos de una nueva alfabetización. Comunicar, (24), 77-84. Recuperado de https://www.redalyc.org/ pdf/158/15802412.pdf

Fernández, F. J., Fernández, M. J., y Rodríguez,J.M.(2018).Diseñoyvalidación de un instrumento de medida del perfil de formación docente en tecnologías de la información y comunicación. Revista Española de Pedagogía, 76(270), 247270. Recuperado de https://www.jstor. org/stable/26547070

Fernández, F. J., y Fernández, M. (2016). Los docentes de la Generación $\mathrm{Z}$ y sus competencias digitales. Comunicar, 46(24), 97-105. doi: http://dx.doi. org/10.3916/C46-2016-10

Fernández, J. C., Fernández, M.C., y Cebreiro, B. (2016). Desarrollo de un cuestionario de competencias en TIC para profesores de distintos niveles educativos. PixelBit: Revista de Medios y Educación, 48, 135-148. doi: http://dx.doi.org/10.12795/ pixelbit.2016.i48.09 
García, A., Arras, A. M., González, L. M., Hernández, A., Martín, J., Prada, S., Tejedor, F. J., Fierro, L. E., y Torres, C. A. (2011). Competencias en TIC y rendimiento académico en la universidad: Diferencias por género. Recuperado de https://gredos.usal. es/jspui/bitstream/10366/121354/1/ libro\%20competencias\%20en\%20 TIC $\% 2$ oy $\% 2$ orendimiento $\% 20$ academico\%20universidad.pdf

García, F., Portillo, J., Romo, J., y Benito, M. (2007). Nativos digitales y modelos de aprendizaje. SPDECE, 1-11. Recuperado de https://www.businessintelligence. info/assets/varios/nativos-digitales.pdf

García, R., Rebollo, Á., y García, C. (2016). Relación entre las preferencias de formación del profesorado y su competencia digital en las redes sociales. Bordón. Revista de pedagogía, 68(2), 137-153. doi: https://doi.org/10.13042/ Bordon.2016.68209

González, J., Espuny, C., de Cid, M. J., y Gisbert, M. (2012). INCOTIC-ESO. Cómo autoevaluar y diagnosticar la competencia digital en la Escuela 2.0. Revista de Investigación Educativa, 3o(2), 287-302. Recuperado de https://revistas.um.es/rie/article/ download $/ 200571 / 163361 / \#$ page $=47$

Hargittai, E., y Hinnant, A. (2008). Digital Inequality: Differences in Young Adults' Use of the Internet. Communication Research, 35, 602-621. Recuperado de https://www.researchgate. net/profile/Amanda Hinnant/ publication/249683187 Digital InequalityDifferences in Young Adults\%27 Use of the Internet/ links/55d7486408aec156b9aa0a58.pdf

Hernández, R., Fernández, C., y Baptista, P. (2007). Metodología de la investigación ( $4^{\mathrm{a}}$. ed.). México: McGraw-Hill.

Inche, J., Andía, Y., Huamanchumo, H., López, M., Vizcarra, J., y Flores, G. (2003). Paradigma cuantitativo: un enfoque empírico y analítico. Industrial data, 6(1), 23-37. Recuperado de https:// www.redalyc.org/html/816/81606104/

INTEF (2017). Marco Común de la Competencia Digital Docente. Madrid: Ministerio deEducación Cienciay Deporte. Recuperado de https://aprende.intef.es/ sites/default/files/2018-05/2017 1020 Marco-Com\%C3\%BAn-de-CompetenciaDigital-Docente.pdf

ISTE, International Society of Technology in Education (2008). Versión traducida del informe de los estándares nacionales de Estados Unidos de Tecnologías de la Información y la Comunicadión para docentes. Recuperado de https://id.iste. org/docs/pdfs/nets-for-teachers-2008 spanish.pdf?sfvrsn=2n

Kachouie, S., y Villanueva, C. C. (2018). Evolución y Perspectivas de la brecha digital en la Unión Europea. Revista ComHumanitas, 9(2), 101-115. doi: https://doi.org/10.31207/rch.vgi2.168

Krumsvik, R. (2008). The emerging digital literacy among teachers in Norway. The story of one digital literate teacher. En R. Koboyashi, New Educational Technology (105-155). New York: Ed. Nova Science Publishers, Inc.

Krumsvik, R. J. (2011). Digital competence in the Norwegian teacher education and schools. Högre utbildning, 1(1), 39-51. Recuperado de https:// hogreutbildning.se/index.php/hu/ article/download/874/1817

Ley Orgánica 2/2006, de 3 de mayo, de Educación. Boletín Oficial del Estado, 4 de mayo de 2006, 17158-17207. Recuperado de https://www.boe.es/ buscar/pdf/2006/BOE-A-2006-7899consolidado.pdf

Ley Orgánica 8/2013, de 9 de diciembre, para la Mejora de la Calidad Educativa. Boletín Oficial del Estado, 10 de diciembre de 2013, 97858- 97921. Recuperado de https://www.boe.es/buscar/pdf/2013/ BOE-A-2013-12886-consolidado.pdf 
Livingstone, S., y Helsper, E. (2007). Gradations in digital inclusion: children, young people and the digital divide. New Mediay Society, 9(4), 671-696.doi: http:// dx.doi.org/10.1177/1461444807080335

Lozoya, S. M., Cuervo, A. V., Armenta, J. A., López, R. I. G., y Salazar, O. C. (2013). Competencias digitales en docentes de educación secundaria. Municipio de un Estado del Noroeste de México. Perspectiva Educacional, formación de profesores, 52(2), 135-153. Recuperado de https://www.redalyc.org/ pdf/3333/333328170007.pdf

Martín, D., Sáenz, M., Santiago, R., y Chocarro, E. (2016). Diseño de un instrumento para evaluación diagnóstica de la competencia digital docente: formación flipped classroom. Revista Didáctica, Innovación y Multimedia, DIM, 33, 1-15. Recuperado de https:// www.raco.cat/index.php/DIM/article/ viewFile/306791/396783

Mishra, P., y Koehler M. J. (2006). Technological pedagogical content knowledge: A framework for teacher knowlege. Teachers college record, 108(6), 1017-1054. Recuperado de http:// citeseerx.ist.psu.edu/viewdoc/download?doi=10.1.1.523.3855\&rep $=\mathrm{rep} 1 \&-$ type $=$ pdf

Mishra, P., y Koehler M. J. (2008). Introducing TPCK. En AACTE Committee on Innovation and Technology (Ed.), Handbook of Technological Pedagogical Content Knowledge for Educators. New York: Routledge. Recuperado de http:// punyamishra.com/

Ortega, V. (1 de mayo de 2011). Maestros: Autorretrato. Nexos en línea. Recuperado de https://www.nexos.com.mx/?p=14272

Pérez, A., y Rodríguez, M. J. (2016). Evaluación de las competencias digitales autopercibidas del profesorado de Educación Primaria en Castilla y León (España). Revista de Investigación
Educativa, 34(2), 399-415. doi: http:// dx.doi.org/10.6018/rie.34.2.215121

Prensky, M. (2001). Nativos digitales, inmigrantes digitales. On the horizon, 9(5), 1-7. Recuperado de http://files. educunab.webnode.cl/2000000625 aba35bb22/Nativos-digitales-parte1.pdf

Prensky, M. (2011). Enseñar a Nativos Digitales. Madrid: SM.

Real Decreto 1105/2014, de 26 de diciembre, por el que se establece el curriculum básico de la Educación Secundaria Obligatoria y el Bachillerato, Boletín Oficial del Estado, 3 de enero de 2015, 169-546. Recuperado de https://www.boe.es/boe/ dias/2015/01/03/pdfs/BOE-A-2015-37. pdf

Real Decreto 1147/2011, de 29 de julio, por el que se establece la ordenación general de la formación profesional del sistema educativo. Boletín Oficial del Estado, 30 de julio de 2011, 1-34. Recuperado de https://www.boe.es/buscar/pdf/2011/ BOE-A-2011-13118-consolidado.pdf

Real Decreto 126/2014, de 28 de febrero, por el que se establece el currículo básico de la Educación Primaria. Boletín Oicial del Estado, 1 de marzo de 2014, 19349-19420. Recuperado de http:// www.juntadeandalucia.es/averroes/ centros-tic/04006151/helvia/aula/ archivos/repositorio/250/389/Real Decreto 126-2014 de 28 de febrero. pdf

Red XXI Educacyldigital (2010). Plan de formación del profesorado para el desarrollo y perfeccionamiento de la competencia digital. Consejería de educación, Dirección General de Calidad, Innovación y Formación del Profesorado. Junta de Castilla y León. Recuperado de http://csfp.centros.educa.jcyl.es/sitio/ upload/Modelo de Competencias Profesionales del Profesorado Definitivo JCyL.pdf

Riascos, S., Ávila, G., y Quintero, D. (2012). Las TIC en el aula: percepciones de 
los profesores universitarios. Revista Educación y Educadores, 12(3), 133-157.

Román, S., y Almansa, A. (2016). Adultos y mayores frente a las TIC. La competencia mediática de los inmigrantes digitales. Comunicar, 24(49), 101-109. doi: http:// dx.doi.org/10.3916/C49-2016-10

Romero, L. N., Salinas, V., y Mortera, F. J. (2010). Estilos de aprendizaje basados en el modelo de Kolb en la educación virtual. Apertura: Revista de innovación educativa, 2(1), 72-85. Recuperado de https://dialnet.unirioja.es/descarga/ articulo/5547097.pdf

San Nicolás, M. B., Fariña, E., y Area, M. (2012). Competencias digitales del profesorado y alumnado en el desarrollo de la docencia virtual. El caso de la Universidad de La Laguna. Revista Historia de la Educación Latinoamericana, 14(19), 227-245. doi: http://dx.doi.org/10.9757/Rhela.19.10

Serrano A., y Martínez, E, (2015). La Brecha Digital: Mitos y Realidades. México: UABC. Recuperado de http://www. labrechadigital.org

Shulman, L. S. (1986). Those who understand: Knowledge growth in teaching. Educational researcher, 15(2), 4-14. Recuperado de https://www.jstor. org/stable/pdf/1175860.pdf

Tapasco, O. A., y Giraldo, J. A. (2017). Estudio Comparativo sobre Percepción y uso de las TIC entre Profesores de Universidades Públicas y Privadas. Formación universitaria, 10(2), 03-12. doi: http://dx.doi.org/10.4067/So71850062017000200002
UNESCO (2005). Hacia las sociedades del conocimiento. París: Ediciones UNESCO. Recuperado de http://unesdoc.unesco. org/images/0014/001419/141908s.pdf

UNESCO. (2008). Estándares de competencia TIC para docentes. París. Recuperado de https://www.oei.es/tic/ UNESCOEstandaresDocentes.pdf

UNESCO. (2011). UNESCO ICT Competency Framework for Teachers. Paris. Recuperado de https://iite.unesco.org/ pics/publications/en/files/3214694.pdf

Valdés, A., Arreola, C., Angulo, J., Carlos, E., y García, R. (2011). Actitudes de docentes de educación básica hacia las TIC. Magis. Revista Internacional de Investigación en Educación, 3(6), 379392. Recuperado de https://www.redalyc. org/pdf/2810/281021734008.pdf

Vallejo, P. M. (2012). Tamaño necesario de la muestra: ¿Cuántos sujetos necesitamos? Estadística aplicada, 24(1), 22-39. Recuperado de http://www. academia.edu/download/31398804/ Tamanomuestra-INTERESANTE.pdf

Vázquez, E. (2015). El reto de la formación docente para el uso de dispositivos digitales móviles en la educación superior. Perspectiva Educacional, Formación de Profesores, 54(1), 149-162. doi: http:// dx.doi.org/10.4151/07189729-Vol.54Iss.1-Art.236

Vera, J. A., Torres, L. E., y Martínez, E. E. (2014). Evaluación de competencias básicas en TIC en docentes de educación superior en México. Píxel-Bit. Revista de Medios y Educación, (44), 143155. doi: http://dx.doi.org/10.12795/ pixelbit.2014.i44.10 


\section{PERFIL ACADÉMICO Y PROFESIONAL DE LOS AUTORES}

Juan Luis Cabanillas García. Doctorando de la Universidad de Extremadura en la línea de investigación de innovación en formación del profesorado, asesoramiento, análisis de la práctica educativa y TIC en educación. Líneas de investigación: Nuevos entornos virtuales de educación y calidad de la enseñanza virtual.

E-mail: jucabanil@alumnos.unex.es

Ricardo Luengo González. Coordinador del Grupo de Investigación CIBERDIDACT, miembro de la Sociedad Española de Investigación en Educación Matemática. Coautor de numerosos libros y artículos científicos, ha dictado conferencias en varios congresos en Argentina, Venezuela, Portugal y España, así como ha participado en numerosos proyectos de investigación. Línea de investigación: matemáticas y nuevas tecnologías.

E-mail: rluengo@unex.es

Jose Luis Torres Carvalho. Profesor de Educación Primaria en el Agrupamento de Escuelas $\mathrm{n}^{\circ} 1$ de Elvas y profesor asociado del Departamento de Didáctica de las Ciencias Experimentales y Matemáticas de la Facultad de Educación de la Universidad de Extremadura. Fue asistente convidado del Departamento de Pedagogia e Educação de la Universidade de Évora - Portugal y profesor destacado en su Centro de Competencia TIC. Línea de investigación: Didáctica de las matemáticas. E-mail: jltc@unex.es

Dirección:

Facultad de Educación

Universidad de Extremadura

Avenida de Elvás s/n

Badajoz (España)

Fecha de recepción del artículo: 28/03/2019

Fecha de aceptación del artículo: 15/07/2019

Fecha de aprobación para maquetación: 13/10/2019 